\title{
An Emergency Energy Management System for Microgrid Restoration after Blackout
}

\author{
Edoardo De Din, Charukeshi Joglekar, Gianluca Lipari, Ferdinanda Ponci, Antonello Monti \\ Institute for Automation of Complex Power Systems - RWTH Aachen University \\ Aachen, Germany \\ ededin,cjoglekar,glipari,fponci,amonti@eonerc.rwth-aachen.de
}

\begin{abstract}
The increase of installed renewable resources and energy storage systems could deeply change the black-start procedure, from the classical top-down to a microgrid-based approach. In this sense, an Emergency Energy Management System (EEMS) is developed to the isolated grid that results after the black-out to optimize the restoration process. The proposed EEMS coordinates the elements of the microgrid in order to provide the required electrical power to critical loads, managing the State of Charge (SOC) of the batteries and the available power from the remaining resources. The proposed approach is tested with a Real Time Digital Simulator (RTDS).
\end{abstract}

Index Terms-Energy Management System, Blackout, Microgrid, Optimal

\section{INTRODUCTION}

The outage of some critical loads such as communication infrastructure, data centres or water distribution during a blackout can heavily affect the essential community services. Providing electrical power to these critical loads represents the main goal of the restoration procedure. The restoration could make use of the backup diesel generators already installed in the LV electrical grid [1] that should provide electricity to a building or a group of buildings in the emergency situation. This generators could directly be installed in the building and continuously maintained or they could be sent in the remote area when the blackout happen [2]. Another possible approach could be the use of the ever increasing presence of Distributed Energy Resources (DERs) in the distribution networks.

This second approach has the advantage of using the resources that may be already installed in the distribution grid and could eliminate the cost of maintaining backup diesel generators. As described in [3] the use of the distributed resources in the distribution grid could positively impact the recovery of energy supply during power outages. In this scenario, a portion of the distribution grid characterized by a sufficient number of DERs could eventually operate as an island if designed to do so [4], forming the so-called isolated microgrid. Here the loads are not individually fed by the backup generators but they are interconnected in the isolated grid.

In this direction, some studies have defined black-start procedures with the available resources of the distribution grid [5] [6], in particular applying optimization algorithms [7] [8]. In [7] the authors have focused on maximizing load restoration without considering the role of the DERs, whereas in [8] the use of the Energy Storage Systems (ESSs) is considered but limited to a single unit, moreover coordination of different resources and ESSs for performing voltage control is not taken into consideration.

The proposed EEMS implements the control of the State Of Charge (SOC) discharging rate of the ESSs, realized with distributed voltage and frequency control strategies, by modifying the coefficients governing the consensus rule. Moreover, it optimizes the other distributed resources utilization, in order to reduce the power provided by the ESSs while maintaining the voltage within the limits. Hence, the EEMS calculates an optimal solution and verifies the voltage constraints and the power flow equations.

Therefore the EEMS is applied to maintain the islanded grid in a stable condition for the longest possible time, given that the time for restoration of the main grid can not be predicted.

\section{The Emergency Energy Management System}

The proposed approach implements a centralized EEMS that manages the installed distributed generators and storage systems of the isolated grid during the power outage and calculates the most suitable configuration of the isolated microgrid to provide the power in blackout scenario. The algorithm remotely controls and selects the switches of the street cabinets to disconnect or connect a portion of the LV grid containing loads and DERs.

In order to simplify the calculation, the loads and DERs are seen as aggregated elements at the point of interconnection with the street cabinets [9] [10]. Therefore, this approach do not actively control the loads and the generators individually but rather acts on the aggregation of them. Hence, this approach does not maximize the flexibility because the reconnection of the street cabinets implies the re-connection of all the connected loads. However, in this way the problem is less complex. Moreover this approach may be immediately applied in the remote control of the street cabinets, without requiring direct load control infrastructure. Before the blackout occurs, the number of available resources, the values of the State Of Charge (SOC) of the batteries and the amount of power consumption is constantly sent to the EEMS and stored. This information is used in the blackout restoration procedure to perform the start up algorithm, as described in 1 .

During the blackout and before starting the re-connection procedure, the EEMS calculates an optimal solution based on the last information stored, able to provide the sufficient amount of power to the critical loads. During the blackout the

(c) (c) 2018 IEEE. Personal use of this material is permitted. Permission from IEEE must be obtained for all other uses, in any current or future media, including reprinting/republishing this material for advertising or promotional purposes, creating new collective works, for resale or redistribution to servers or lists, or reuse of any copyrighted component of this work in other works. 


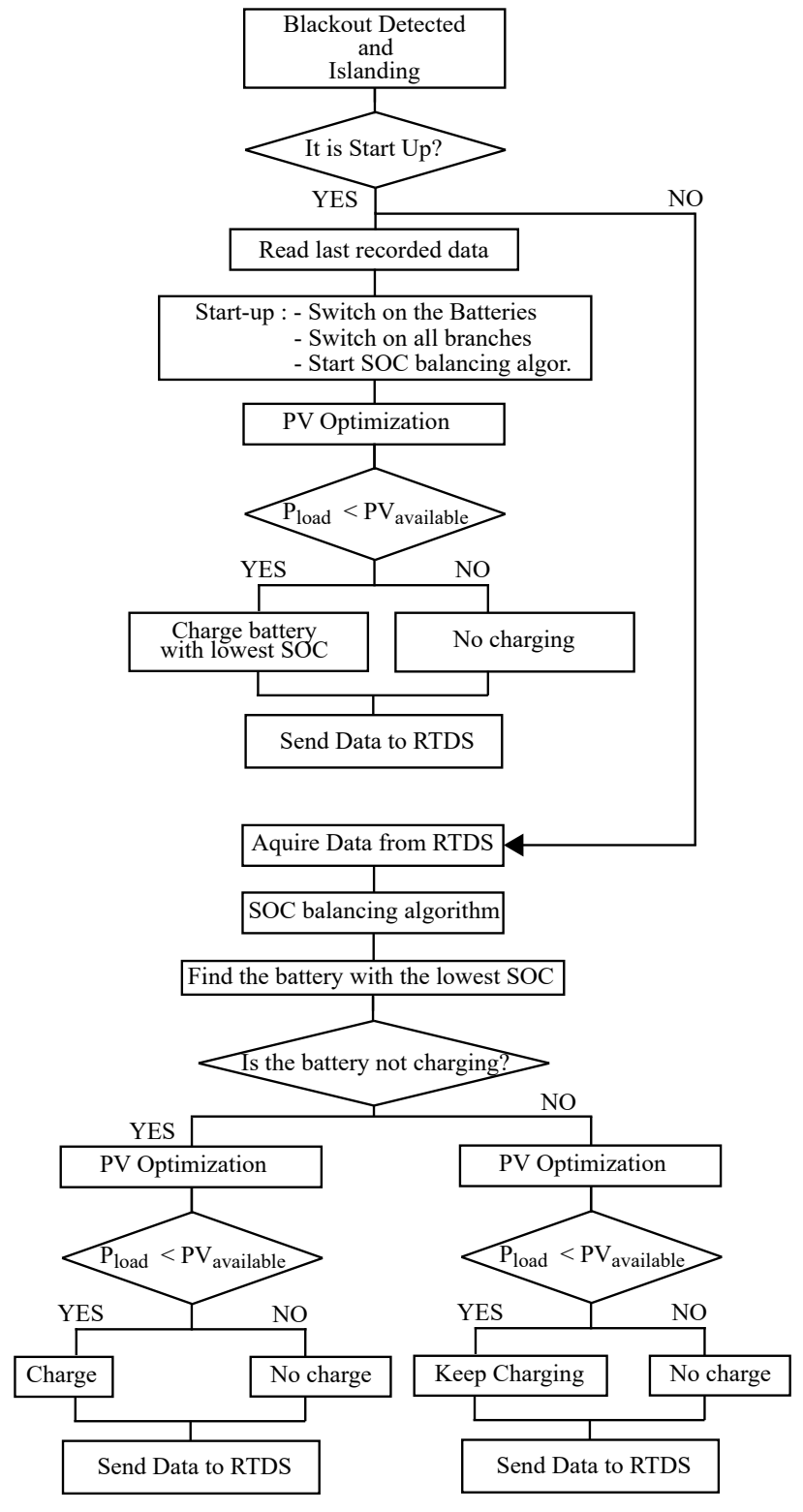

Fig. 1: Flowchart of the proposed EEMS

EEMS calculates the grid configuration that should guarantee a stable configuration in term of voltage requirements, the black start process is initiated with the reconnection of the ESSs, performing the distributed voltage control [11], as described in [8]. Once the microgrid has achieved a steady-state operation, the DERs controlled with active and reactive power set-points, the grid-feeding inverters [12], can be connected [13]. This helps reducing the amount of power provided by the batteries and therefore the discharging time, given that the amount of power provided by the storage systems to support the voltage and feed the loads is consequently reduced. During the time the microgrid remains isolated, the EEMS monitors the SOC of the batteries and the available power of the other resources. To maintain uninterrupted isolated configuration for the longest possible time, the EEMS must guarantee a certain number of energy storages connected with a sufficient value of SOC. Therefore, the grid configuration can be constantly changed to connect the charged batteries of an aggregated system and recharge the discharged batteries connected to a different $\mathrm{SC}$ with the power generated by the DERs. Moreover, whenever a new configuration is defined, the EEMS must verify if the new configuration is optimal, as described in 1 . In the proposed approach when the results of the optimization process shows that the amount of load in one aggregated system is too high, the complete aggregated system is disconnected.

Therefore, the complete EEMS approach is composed of two different algorithms - Distributed Secondary Control for the SOC balancing and the Optimization algorithm for the connection of the PVs and the ESSs charging - representing two different aspects of the procedure. As described in Figure 1 , if the blackout is detected the algorithm begins the start up procedure. In this case all the ESSs, which are connected to voltage-controlled voltage source inverters (VCVSIs), perform the restoration of the voltage and frequency in the islanded microgrid. Once the VCVSis are connected, the algorithm for balancing the SOC of the connected batteries is started. Moreover, the optimization algorithm is performed to verify if there is enough power available from the other grid-feeding inverters (the PVs in the proposed scenario) to start recharging the battery with the lowest SOC. In any case, the optimization algorithm must always guarantee that the charging procedure does not affect the support of the loads - especially if some critical loads are presents.

When the start up procedure is finished, the algorithm enters in a loop where the data are constantly acquired and used to perform the different steps of the EEMS. This assumption requires a functioning communication system after the restoration procedure that could be achieved by applying a load prioritization or by installing a dedicated communication architecture based on powerline communication [14]. The details of the algorithms used of the SOC balancing and the optimization are described in the following Subsections.

\section{A. Distributed Secondary Control for the SOC balancing}

The distributed secondary control based on the consensus theory is applied to solve the problem of voltage and frequency control combined with the active and reactive power sharing in a microgrid where voltage-controlled voltage-source inverters (VCVSIs) and current-controlled voltage-source inverters (CCVSIs) are both present. In the proposed scenario the VCVSIs are considered to be the energy storage systems (ESSs) interfaced with an inverter, whereas the the CCVSIs are considered to be the PV systems. Therefore in this configuration, the primary controller of the VCVSIs sets the output voltage and frequency to the nominal reference [12] and the primary controller of the CCVSIs controls the active and reactive power injections. As described in [15], the converters that are used to perform the secondary distributed control are the VCVSIs. 

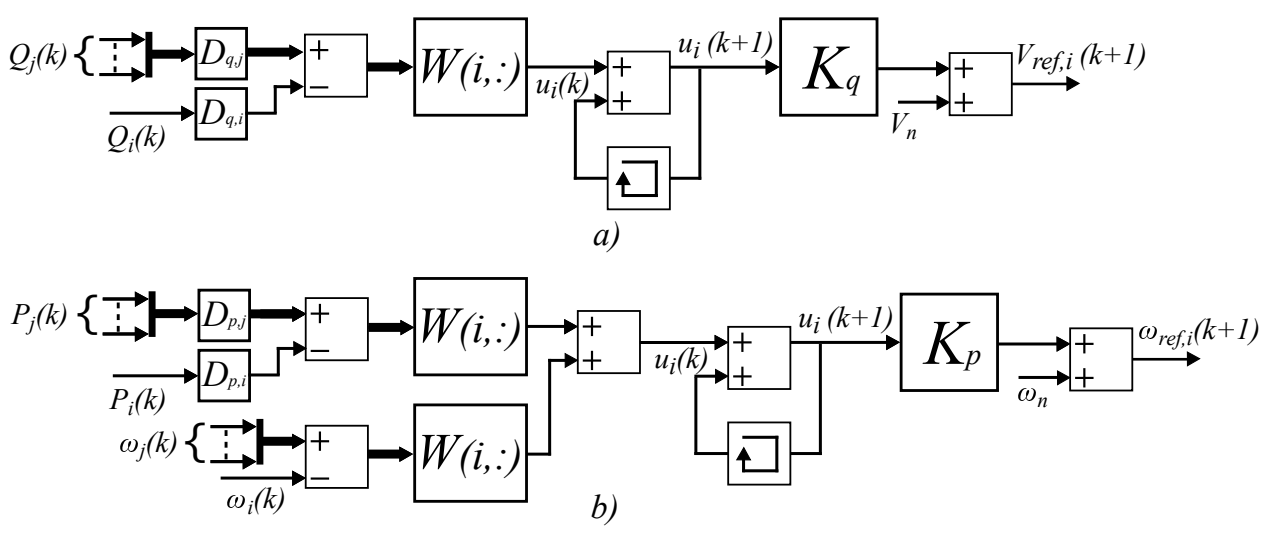

Fig. 2: Distributed Secondary Control: a) Voltage / Reactive Power, b) Frequency / Active Power

In order to parallel connect VCVSIs to the same feeder, the droop control has been extensively used [12], however it has been proven not able to achieve a desired reactive power sharing [11]. The consensus-based control described in [16] has been applied in [11] to perform accurate power sharing in a distributed manner avoiding the use of the droop control. Moreover, the control does not heavily affect the voltage levels at different nodes if the control parameters are selected within a meaningful range that can be evaluated as described in [11].

\section{- Voltage / Reactive Power Control:}

The consensus-based reactive power sharing and voltage control is based on the algorithm described in [11]. The algorithm in its discrete formulation can be described as follows:

$$
\begin{array}{ll}
V_{r e f}(k+1) & =V_{n}+K_{q} u(k+1) \\
u(k+1) & =u(k)+W D_{q} Q(k)
\end{array}
$$

where, the different elements of the equations are:

$K_{q}:=\operatorname{diag}\left(k_{i}^{q}\right), D_{q}:=\operatorname{diag}\left(n_{i}^{q}\right), W:=-L$

The variable $V_{r e f}(k+1)$ represents the control outputs vector of the distributed control that are used as voltage references value for the primary controllers of the VCVSIs [11]. This is obtained with the sum of the nominal voltage value $V_{n}$ and the output of the consensus equation, where the matrix $K$ is used to modify the convergence speed of the algorithm. In order to achieve the consensus, the matrix $W$ is set to be the negative of the matrix $L$, which is the Laplacian of the communication graph [16]. The matrix $D_{q}$ contains the sharing coefficients for the reactive power and it multiplies the vector $Q$, representing the measured reactive power generated by the converters. Therefore the consensus among the nodes participating on the distributed control is achieved by applying the discrete integration of the difference between the elements of $Q$ based on the communication links.

\section{- Frequency / Active Power Control:}

The consensus-based of the active power sharing control follows the same concept previously described in the voltage control and aims at the desired sharing together whereas controlling the frequency. The algorithm is based on the distributed cooperative frequency control described in [15], where the power sharing among the communicating nodes can be eventually modified by changing the coefficients of the control equations. The equations describing the control algorithm are:

$$
\begin{array}{ll}
\omega_{r e f}(k+1) & =\omega_{n}+K_{p} u(k+1) \\
u(k+1) & =u(k)+W \omega(k)+W D_{p} P(k)
\end{array}
$$

where, the different elements of the equations are:

$K_{p}:=\operatorname{diag}\left(k_{i}^{p}\right), D_{p}:=\operatorname{diag}\left(m_{i}^{p}\right), W:=-L$

The reference values for the primary controllers $\omega_{\text {ref }}(k+$ 1) are calculated based on the nominal value $\omega_{n}$ and the output of the consensus algorithm. The consensus algorithm is based on the same communication graph $L$ previously described (and the same $W$ ), where in this case the difference between the values of the measured $\omega$ and $P$ are digitally integrated. Both the Voltage / Reactive Power Control and the Frequency / Active Power Control are described in Figure 2

In order to set the sharing coefficients of the active power injection of the different VCVSIs, an algorithm has been developed that modifies in real time the values of $D_{p}$ to balance the SOC of the batteries. The proposed algorithm aims at reducing the discharging rate of the battery with the lower initial value of the SOC. By doing so, the algorithm keeps the storage systems connected for as long as possible if there is no sufficient $\mathrm{PV}$ available to disconnect at least one of them, increasing the stability of the microgrid. This is clear from the fact that loosing one VCVSI results in removing one node dedicated to voltage control.

The change of the sharing coefficients follows a simple 


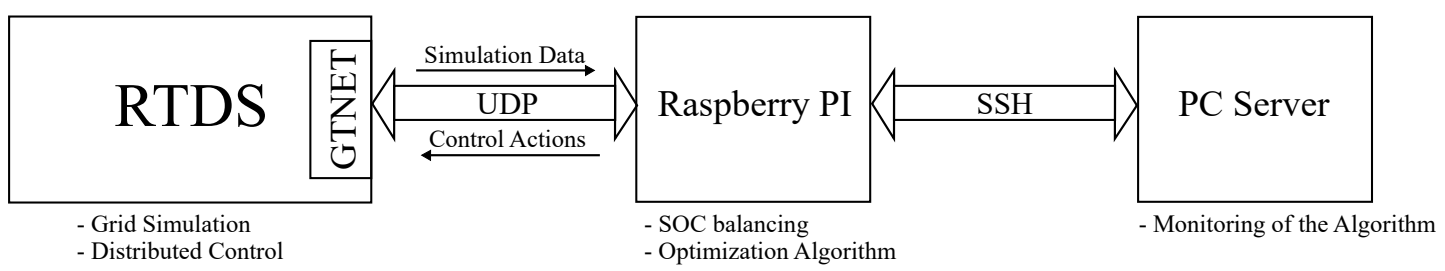

Fig. 3: Experimental Setup

algebraic rule that correlates the coefficients among them. In the proposed approach, at the first instant all the coefficients are equal and therefore the sharing is identical. The algorithm uses the initial value as a reference for modifying the contribution of each node. Therefore, at each time the new set of sharing coefficients are calculated as follows:

$$
\begin{array}{ll}
m_{i}^{p}(k) & =m_{\text {init }}^{p} / S O C_{\text {ratio }}(k) \\
S O C_{\text {ratio }}(k) & =\frac{S O C_{i}(k)}{S O C_{\max }(k)}
\end{array}
$$

where $m_{\text {init }}^{p}$ is the initial value of the sharing coefficient and the $S O C_{\text {ratio }}(k)$ describes the ratio between the i-th $\mathrm{SOC}$ values $\left(S O C_{i}(k)\right)$ and the highest value $\left(S O C_{\max }(k)\right)$ at the instant $k$.

The resulting coefficients are sorted from the smallest to the largest value and are sent in the reverse order to the secondary controllers of the EESs, which are sorted based on the measured value of their SOC. This means that the smallest coefficient is applied to the secondary controller of the power converter with the higher SOC status. With the application of the algorithm, the converter with the higher SOC provides more power and gradually the other converters provide less power output.. The application of this algorithm allows the progressive balance of the SOCs of the energy storages.

\section{B. Optimal connection of the PVs and EESs charging}

Once the voltage has been restored by means of the ESSs, the PVs installed in the aggregated system could be used to reduce the amount of power provided by the batteries - and therefore reducing the discharging rate - while maintaining the voltage of the different nodes within certain limits as described in Figure 1. The calculation of the active and reactive power set points for the CCVSIs is performed with the purpose of optimizing the power flow in the microgrid while maintaining the voltage in the limits. The optimization algorithm tries to minimize the active and reactive power losses maintaining the voltage deviation within the typical allowed voltage deviation of $5 \%$ [17] of the nominal value and considering the power flow equations as constraints. Therefore the objective function can be described with the following equations:

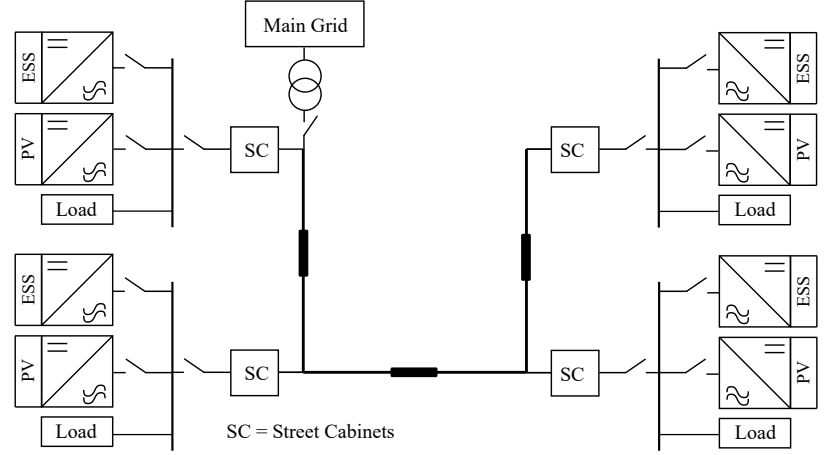

Fig. 4: Simulated LV distribution grid

$$
\begin{aligned}
& \underset{P_{\text {loss }}, Q_{\text {loss }}}{\operatorname{minimize}} \quad\left(P_{\text {loss }}+Q_{\text {loss }}\right)^{\frac{1}{2}} \\
& P_{\text {loss }}=\sum P_{P V}+\sum P_{E S S}-\sum P_{\text {demand }} \\
& Q_{\text {loss }}=\sum Q_{P V}+\sum Q_{E S S}-\sum Q_{\text {demand }}
\end{aligned}
$$

where total active and reactive power losses $-P_{\text {loss }}$ and $Q_{\text {loss }}$ - are calculated as the sum of the total active and reactive power sharing that could be available from the PVs $-P_{P V}$ and $Q_{P V}$ - and the active and reactive power generated by the EES - $P_{E E S}$ and $Q_{E E S}$ - reduced by the total load power demand - $P_{\text {demand }}$ and $Q_{\text {demand }}$. The results of minimizing the losses reduces the the power exchange between the aggregations, but also tries to make use of all the available power in case of a sudden increase of a load in one of the aggregation (e.g. the reconnection of one battery in charging mode). This leads to efficient grid operation, because the losses are reduced and the use of the PVs are optimized.

The application the optimization algorithm results in a reduction of the power output of the battery, given that optimized values for the PVs help supporting the voltage at the nodes. Moreover, the algorithm monitors the PVs available power to verify if it could be sufficient to start re-charging one battery. Therefore, a new optimization is performed increasing the load value at the node where the battery should be recharged.

\section{EXPERIMENTAL SETUP}

The purpose of the experimental setup is to test the capability of the proposed strategy to find an optimal solution able to provide power to the microgrid in the restoration process and to maintain it while the SOC of the storage systems, the total absorbed power and the generated power dynamically 


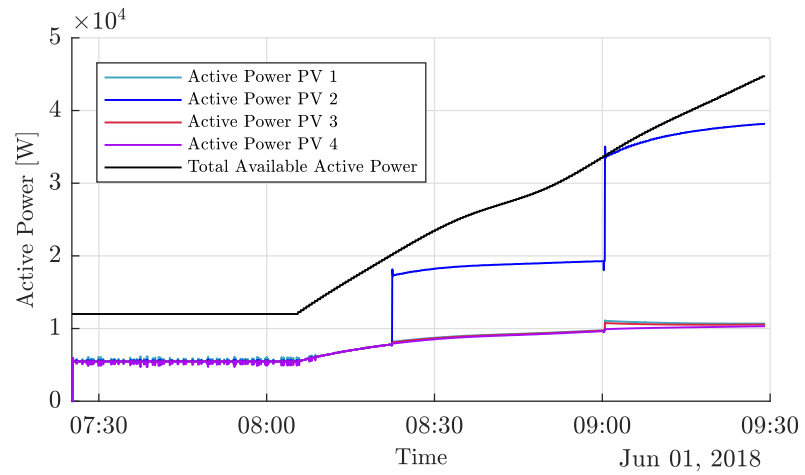

(a) Active Power Generated by the PV

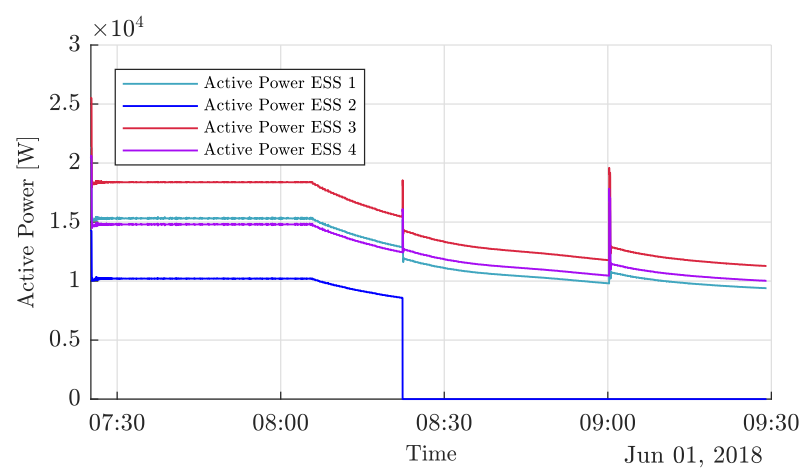

(b) Active Power Generated by the ESSs

Fig. 5

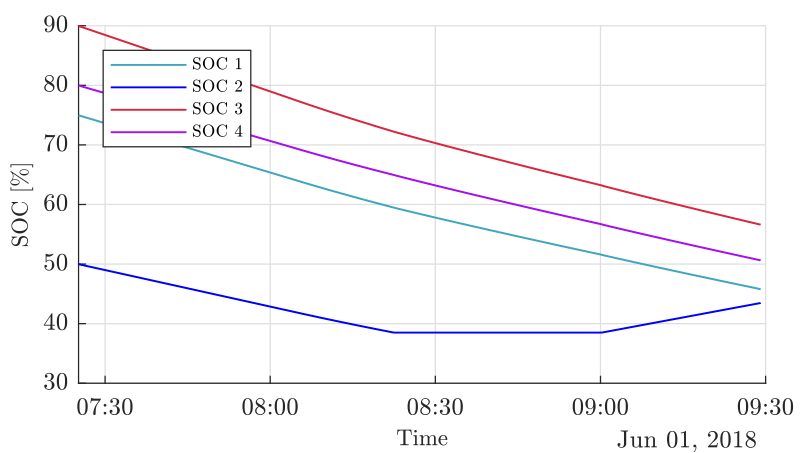

(a) SOC of the ESSs

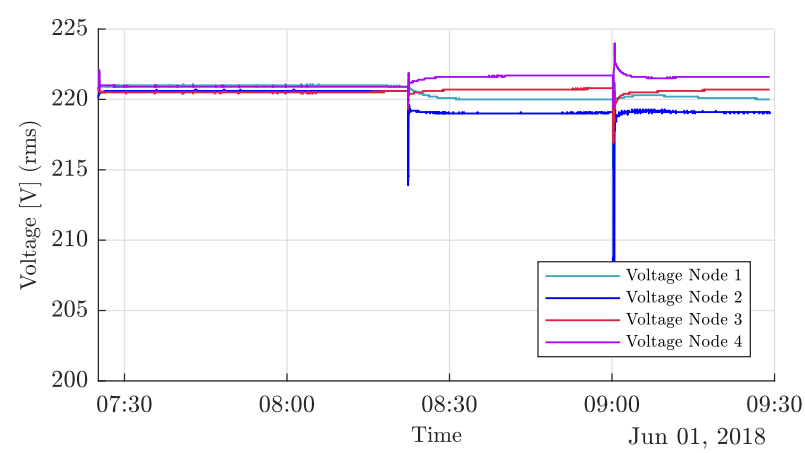

(b) Voltages at the nodes of the Microgrid

Fig. 6

change. The test compares the discharging rate of the SOC of the batteries and evaluates if the algorithm is able to detect when a certain battery should be disconnected or recharged based on the available power from the PVs.

In the experimental setup the EEMS is tested on a Low Voltage (LV) distribution grid composed of a feeder where the distribution street cabinets (SC) are assumed to be interconnected via powerline communication (PLC) to guarantee the data exchange between the DERs and the central intelligence also during power outage [14]. Every aggregated electrical node consists of a CCVSI, representing the overall photovoltaic (PV) production for a specific group of houses connected to a node, a battery connected to a VCVSI, representing the amount of energy storage at the node, and a dynamic load that aggregates all the absorbed power at the node. The complete simulated LV distribution grid is described in Figure 4.

The experimental test is performed in Hardware in the Loop (HiL) which has the adavtage of interfacing a real time simulation with physical hardware devices. This architecture allows the test of the proposed algorithm running in an hardware platform and interfacing to the grid (simulated) with the communication protocol.

In the test, the LV grid is simulated in the Real Time Digital Simulator (RTDS) with a time step of $150 \mu$ s and the results of the real-time simulation are sent by means of UDP protocol with a GTNET - Network interfaced card to a Raspberry
PI. The equations of the distributed control are implemented in the RTDS and the sharing coefficients are received from the Raspberry PI, which performs the algorithm for the SOC balancing as described in Figure 1.

The calculation of the topology reconfiguration and the optimal set-points for the PV generation is calculated in the Raspberry PI, as described in Figure 3. At each new calculation, the device receives the new measured vales of generated and load powers, voltage and angles, and based on them it calculates the optimal solution. The optimization algorithm is performed by means of the open-source optimization solver IPOPT (Interior Point OPTimizer) [18] and the Python-based open-source optimization modeling language PYOMO [19], which allows high-level programming language to interface with the optimization solver.

\section{REsults}

This section presents the results of the proposed architecture applied on a simulated blackout scenario in a LV grid characterized by aggregated systems as described in Figure 4 . The microgrid, disconnected from the main grid, is composed of four nodes where a street cabinet connects the aggregation of the ESSs, the PVs and loads.

The total load for each aggregation is $20 \mathrm{~kW}$ and it does not change during the simulation. In the simulated scenario, the ESS aggregation is supposed to absorb a constant power 
of $20 \mathrm{~kW}$ during the recharging mode. Therefore, when the aggregation is charging, the power of the total aggregated load doubles.

The PV profile, describing the maximum available power, follows an ascending characteristic from $12 \mathrm{~kW}$ to $44.7 \mathrm{~kW}$ within two hours. Between 07:25 and 8:05 the PV profile remains stable to $12 \mathrm{~kW}$ and then consequently also the power generated by the aggregation of the PVs. In this configuration, the available power from the PVs is not enough to permit a disconnection of one of the ESS aggregation, therefore the optimization algorithm calculates a value for the PVs set-points that is not very high, but sufficient to reduce the power provided by the ESSs as described in Figure 5a. The application of the SOC balancing algorithm determines a different power sharing among the ESSs (Figure 5b), which is the result of modifying the sharing coefficients (as described in Section II). The application of the EEMS in the initial part of the simulation results in a distribution of $75 \%$ of the total generation within the ESSs.

When the PV available power starts increasing, the algorithm calculates new power set-points and consequently the active power generated by the ESSs decreases. A time 08:21 the optimization algorithm verifies that the battery at node $2-$ the one with the lowest SOC - can be disconnected, producing a voltage drop that is rapidly recovered by the distributed control as described in Figure $6 \mathrm{~b}$. In the new configuration, the PV aggregation at node 2 provides most of power necessary to compensate for the battery disconnection whereas the power provided by the other PVs remains limited. As a consequence, the difference between the voltages increases, given that the distributed voltage control looses one regulating node, but the difference is maintained within the $\sim 0.5 \%$.

Finally at time 9:00 the PVs available power is enough to guarantee the ESS aggregation at node 2 to be recharged and consequently the SOC at node 2 starts increasing as described in Figure6a. In this condition the power for the recharge is given by the PVs at node 2, since this configuration is the one that keeps the power losses reduced. After the sudden voltage drop, the voltage deviations at the nodes remain limited.

\section{Conclusion}

The paper has described the implementation of an Energy Management System able to control the resource in an islanded microgrid during a blackout scenario. The EEMS controls the sharing of the ESSs, interacting with the Distributed Secondary control, and optimizes the resources that can provide specific values of active and reactive power. The results have demonstrated that the algorithm is able to share differently the power provided by the batteries and consequently changing the SOC discharging rate of the ESSs. Moreover the algorithm is able to manage the resource available from the PV and decide whether the battery with the lower value of the SOC could be recharged.

One of the first aspects of the proposed EEMS that would be further investigated is the application of the algorithm in a microgrid composed by an higher number of aggregated systems, where it could make sense to distribute the intelligence among different nodes. Moreover, the EEMS should be able to run for a longer time and able to manage the disconnection and reconnection of multiple ESSs.

\section{ACKNOWLEDGMENT}

This work is sponsored by the German Ministry for Industry and Energy (project number 03ET7549A) and the European Unions Horizon 2020 Research and Innovation Programme (Grant Agreement number 700416).

\section{REFERENCES}

[1] J. Feltes and C. Grande-Moran, "Black start studies for system restoration," in Power and Energy Society General Meeting-Conversion and Delivery of Electrical Energy in the 21st Century, 2008 IEEE, pp. 1-8, IEEE, 2008.

[2] J. F. Melley Jr, "Mobile electric power generating systems," Jan. 30 1979. US Patent 4,136,432.

[3] I.-I. E. Commission et al., "Microgrids for disaster preparedness and recovery-with electricity continuity plans and systems," White paper, Geneva, Switzerland, 2014.

[4] "Ieee guide for design, operation, and integration of distributed resource island systems with electric power systems," IEEE Std 1547.4-2011, pp. 1-54, July 2011.

[5] C. Moreira, F. Resende, and J. P. Lopes, "Using low voltage microgrids for service restoration," IEEE Transactions on Power Systems, vol. 22, no. 1, pp. 395-403, 2007.

[6] C. Chen, J. Wang, F. Qiu, and D. Zhao, "Resilient distribution system by microgrids formation after natural disasters," IEEE Transactions on smart grid, vol. 7, no. 2, pp. 958-966, 2016.

[7] C. Yuan, M. S. Illindala, and A. S. Khalsa, "Modified viterbi algorithm based distribution system restoration strategy for grid resiliency," IEEE Transactions on Power Delivery, vol. 32, no. 1, pp. 310-319, 2017.

[8] A. Di Giorgio, A. Giuseppi, F. Liberati, and A. Pietrabissa, "Controlled electricity distribution network black start with energy storage system support," in Control and Automation (MED), 2017 25th Mediterranean Conference on, pp. 781-786, IEEE, 2017.

[9] G. Lipari, G. Del Rosario, C. Corchero, F. Ponci, and A. Monti, "A realtime commercial aggregator for distributed energy resources flexibility management," Sustainable Energy, Grids and Networks, 2017.

[10] T. Morstyn, A. V. Savkin, B. Hredzak, and H. D. Tuan, "Scalable energy management for low voltage microgrids using multi-agent storage system aggregation," IEEE Transactions on Power Systems, 2017.

[11] J. Schiffer, T. Seel, J. Raisch, and T. Sezi, "Voltage stability and reactive power sharing in inverter-based microgrids with consensus-based distributed voltage control," IEEE Transactions on Control Systems Technology, vol. 24, no. 1, pp. 96-109, 2016.

[12] J. Rocabert, A. Luna, F. Blaabjerg, and P. Rodriguez, "Control of power converters in ac microgrids," IEEE transactions on power electronics, vol. 27, no. 11, pp. 4734-4749, 2012.

[13] M. Nuschke, "Development of a microgrid controller for black start procedure and islanding operation," in 2017 IEEE 15th International Conference on Industrial Informatics (INDIN), pp. 439-444, July 2017.

[14] "Powerline at the medium voltage-level." Online at https://www.devolo.com/en/SmartGrid/medium-voltage-level.

[15] A. Bidram, A. Davoudi, F. L. Lewis, and Z. Qu, "Secondary control of microgrids based on distributed cooperative control of multi-agent systems," IET Generation, Transmission \& Distribution, vol. 7, no. 8, pp. 822-831, 2013.

[16] R. Olfati-Saber, J. A. Fax, and R. M. Murray, "Consensus and cooperation in networked multi-agent systems," Proceedings of the IEEE, vol. 95, no. 1, pp. 215-233, 2007.

[17] V. Kekatos, G. Wang, A. J. Conejo, and G. B. Giannakis, "Stochastic reactive power management in microgrids with renewables," IEEE Transactions on Power Systems, vol. 30, no. 6, pp. 3386-3395, 2015.

[18] “Coin-Or - Ipopt." Online at https://projects.coin-or.org/Ipopt

[19] "Pyomo." Online at http://www.pyomo.org/. 